\title{
Paper
}

\section{Some remarks on numerical verification of closed orbits in dynamical systems}

\author{
Tomohiro Hiwaki ${ }^{1 a)}$ and Nobito Yamamoto $^{1}$ \\ 1 The University of Electro-Communications, \\ 1-5-1 Chofugaoka, Chofu, Tokyo, Japan \\ a)hiwaki.realonly@gmail.com
}

Received August 31, 2014; Revised March 19, 2015; Published July 1, 2015

\begin{abstract}
We consider numerical verification methods for existence of a closed orbit in a dynamical system which is described by ODEs. Besides Zgliczyński's method using Poincaré map, the authors proposed a method of verification for closed orbits and their time period. In this paper, we derive a relationship between our method and one of bordering methods which gives some explanation of superiority of this bordering.
\end{abstract}

Key Words: numerical verification, dynamical system, ODEs, closed orbit, Poncaré map, bordering condition

\section{Introduction}

We discuss some relationship among numerical verification methods for existence of a closed orbit in a dynamical system which is described by

$$
\begin{array}{r}
\frac{d \boldsymbol{u}}{d t}=\boldsymbol{f}(\boldsymbol{u}), \quad 0<t<\infty, \\
\boldsymbol{u} \in R^{n}, \quad \boldsymbol{f} \in C^{\infty} .
\end{array}
$$

There are some verification methods which prove that the dynamical system has a periodic solution under some conditions, and specify the periodic time $T^{*}$ and the initial point $\boldsymbol{u}(0)=\boldsymbol{w}^{*}$.

Zgliczyński proposed a method by use of Poincaré map [1]. He needs to identify a return time for each point $\boldsymbol{w}$ on a Poincaré section by which the trajectory starting from $\boldsymbol{w}$ returns the Poincaré section. The authors proposed a method in [2] which uses a projection to the Poincaré section and Newton-like iteration for the time period $T^{*}$, and does not need return time. We refer to the method as HY method. On the other hand, there are simpler methods which solve a two-point boundary value problem with a bordering condition and do not use Poincaré map. The bordering condition can be chosen from various alternatives, among which one special choice seems to give advantageous results in many cases.

In this paper we show that HY method is equivalent to the special choice of bordering condition, which suggests the reason why the choice gives good results. 


\section{2. $C^{1}$ algorithm}

Before discussion of main part, we briefly mention Zgliczyński's method [1]. This contains so called $C^{1}$ algorithm which is adopted in our methods as described below.

Let $P(\boldsymbol{w})$ and $\Gamma$ be a Poincaré map and Poincaré section with $\boldsymbol{w} \in \Gamma$, respectively. For numerical verification of a fixed point of Poincaré map, it is necessary to do validated computation of Poincaré map $P(\boldsymbol{w})$ as well as $\frac{\partial P}{\partial \boldsymbol{w}}(\boldsymbol{w})$.

Define $\varphi(t, \boldsymbol{w})$ as a point at the time $t$ on a trajectory which is a solution of Eq. (1) with the initial condition $\boldsymbol{u}(0)=\boldsymbol{w}$. Then $\frac{\partial P}{\partial \boldsymbol{w}}(\boldsymbol{w})$ is a solution of a variational equation to the system Eq. (1) as follows.

$$
\left\{\begin{array}{l}
\frac{d V}{d t}(t, \boldsymbol{w})=\frac{\partial \boldsymbol{f}}{\partial \boldsymbol{u}}(\varphi(t, \boldsymbol{w})) V(t, \boldsymbol{w}), \\
V(0, \boldsymbol{w})=I
\end{array}\right.
$$

where $V \in R^{n \times n}$ and $I$ is the identity matrix. Specify $t_{w}$ as the return time such that $\varphi\left(t_{w}, \boldsymbol{w}\right) \in \Gamma$ for $\boldsymbol{w}$, then we have $\frac{\partial P}{\partial \boldsymbol{w}}=V\left(t_{w}, \boldsymbol{w}\right)$.

Zgliczyński developed a method of validated computation to verify $\varphi(t, \boldsymbol{w})$ together with $\frac{\partial \varphi(t, \boldsymbol{w})}{\partial \boldsymbol{u}}$ (or $P(\boldsymbol{w})$ together with $\frac{\partial P}{\partial \boldsymbol{w}}$ ), which is called $C^{1}$ algorithm. He called Lohner method for $\varphi(t, \boldsymbol{w}) C^{0}$ algorithm and showed that $C^{0}$ and $C^{1}$ algorithm take essentially the same order of executing time in computation.

A drawback of his method is that he needs a narrow interval including $t_{w}$. In order to get such an interval, he derives a special estimation for $t_{w}$ and use a bisection method.

\section{HY method}

In this section we describe HY method at reader's convenience, as the paper [2] is written by Japanese.

\subsection{Construction of a mapping $Q$}

Let $\tilde{T}$ and $\tilde{\boldsymbol{u}}$ be an approximate period and an approximate trajectory to an closed orbit, respectively, and put $\tilde{\boldsymbol{w}}:=\tilde{\boldsymbol{u}}(0)$. Then we translate the coordinates in order to position $\tilde{\boldsymbol{w}}$ at the origin.

An integral form for $\varphi(t, \boldsymbol{w})$ is derived from Eq. (1) as follows.

$$
\varphi(t, \boldsymbol{w})=\boldsymbol{w}+\int_{0}^{t} \boldsymbol{f}(\varphi(\tau, \boldsymbol{w})) d \tau, \quad 0 \leq t \leq T
$$

Let $\Gamma$ be a plane corresponding to a Poincaré section. The condition in order that $\varphi(T, \boldsymbol{w})$ is settled on $\Gamma$ is

$$
\int_{0}^{T} \boldsymbol{n}^{\dagger} \boldsymbol{f}(\varphi(\tau, \boldsymbol{w})) d \tau=0
$$

where $\boldsymbol{n}$ is the unit normal vector of the plane $\Gamma$, and we choose $\boldsymbol{n}=\frac{\boldsymbol{f}(\tilde{\boldsymbol{w}})}{\|\boldsymbol{f}(\tilde{\boldsymbol{w}})\|}$ using the approximate initial point $\tilde{\boldsymbol{w}}=\mathbf{0}$. Here the letter $\dagger$ means the transpose of a vector.

The mapping $Q$ on $\boldsymbol{z}=\left(\begin{array}{c}T \\ \boldsymbol{w}\end{array}\right)$ is defined as follows.

$$
\begin{aligned}
& Q: \boldsymbol{z}_{0} \rightarrow \boldsymbol{z}_{1}, \\
& \boldsymbol{z}_{0}=\left(\begin{array}{c}
T_{0} \\
\boldsymbol{w}
\end{array}\right), \boldsymbol{z}_{1}=\left(\begin{array}{c}
T_{1} \\
\varphi\left(T_{0}, \boldsymbol{w}\right)
\end{array}\right), \\
& T_{1}=T_{0}-\frac{1}{\gamma} \boldsymbol{n}^{\dagger} \int_{0}^{T_{0}} \boldsymbol{f}(\varphi(\tau, \boldsymbol{w})) \tau .
\end{aligned}
$$


From Eq. (2), the calculation of $T_{1}$ gives Newton iteration to get $T$ such that $\varphi(T, \boldsymbol{w}) \in \Gamma$ when we take $\gamma=\boldsymbol{n}^{\dagger} \boldsymbol{f}(\boldsymbol{w})$. In practical computation, we take $\gamma=\boldsymbol{n}^{\dagger} \boldsymbol{f}(\tilde{\boldsymbol{w}})$ for simplicity which gives quasi-Newton iteration.

Note that a fixed point of the mapping $Q$ gives the time period and the crossing point on $\Gamma$ of a closed orbit of the dynamical system Eq. (1). Therefore a numerical verification of the fixed point of $Q$ proves the existence of a closed orbit of Eq. (1).

The Jacobi matrix of the mapping $Q$ has an eigenvalue equal to 1 , which causes breakdown in computation. To avoid this, we adopt an orthogonal projection to the plane $\Gamma$.

Define a matrix $P_{\Gamma}$ by

$$
P_{\Gamma}=I-\frac{1}{\boldsymbol{n}^{\dagger} \boldsymbol{n}}\left(\begin{array}{c}
0 \\
\boldsymbol{n}
\end{array}\right)\left(0, \boldsymbol{n}^{T}\right)
$$

which projects $\left(\begin{array}{c}T \\ \boldsymbol{u}\end{array}\right)$ to $\left(\begin{array}{l}T \\ \boldsymbol{w}\end{array}\right)$ where $\boldsymbol{w} \in \Gamma$. Note that a fixed point of $P_{\Gamma} Q$ is a fixed point of $Q$ and this corresponds to the period and the crossing point of a closed orbit.

\subsection{Validation of fixed point}

In order to verify a fixed point of the mapping $P_{\Gamma} Q$, we use quasi-Newton method with numerical verification. The Jacobi matrix $Q_{z}$ of $Q$ is written by

$$
Q_{z}(\boldsymbol{z})=\left(\begin{array}{cc}
1-\frac{1}{\gamma} \boldsymbol{n}^{\dagger} \boldsymbol{f}(\varphi(T, \boldsymbol{w})) & \frac{1}{\gamma} \boldsymbol{n}^{\dagger}(I-V(T, \boldsymbol{w})) \\
\boldsymbol{f}(\varphi(T, \boldsymbol{w})) & V(T, \boldsymbol{w})
\end{array}\right),
$$

where $V$ means the derivative of $\varphi$ with respect to $\boldsymbol{w}$ which is a solution of the variational equation to the system Eq. (1).

Let $\tilde{Q}_{z}$ be an approximation to $Q_{z}(\tilde{\boldsymbol{z}})$ with $\tilde{\boldsymbol{z}}=\left(\begin{array}{c}\tilde{T} \\ 0\end{array}\right)$, and define Newton operator by

$$
N(\boldsymbol{z}):=\boldsymbol{z}-\left(I-P_{\Gamma} \tilde{Q}_{z}\right)^{-1}\left(\boldsymbol{z}-P_{\Gamma} Q(\boldsymbol{z})\right)
$$

Take a bounded convex closed set $[Z]=\left(\begin{array}{c}{[T]} \\ {[W]}\end{array}\right)$ constructed by an real interval $[T]$ and an interval vector $[W] \subset \Gamma$ which include the approximation $\tilde{T}$ and $\tilde{\boldsymbol{w}}=\mathbf{0}$, respectively. Then iterate the following.

(1) Check the condition $N([Z]) \subset[Z]$ by interval arithmetic with guaranteed accuracy. If the condition holds, then it is proved that there exists a fixed point of $Q$ within $[Z]$ by Brouwer's fixed point theorem.

(2) If the condition dose not hold, calculate $[Z]:=(1+\varepsilon) N([Z])-\varepsilon N([Z])$ by interval arithmetic and go back to (1). Here $\varepsilon$ is a given small positive number.

In practical computation, we adopt various technique of numerical verification, e.g. Lohner method, mean value form and so on [8]. Application of the mean value form to the operator $N(\boldsymbol{z})$ yields a Krawczyk operator.

For computation of $V$, we adopt $C^{1}$ algorithm by Zgliczyński. Note that a precise return time is not necessary by virtue of Newton iteration for $T$, which is necessary in Zgliczyńsky's method on the other hand.

\section{Bordering method}

The following two-point boundary value problem with a bordering condition is derived from Eq. (1) with Poincaré section $\Gamma$. 


$$
\left\{\begin{array}{l}
\boldsymbol{n}^{\dagger} \varphi\left(T_{b}, \boldsymbol{w}\right)=0 \\
\varphi(T, \boldsymbol{w})=\boldsymbol{w}
\end{array}\right.
$$

Here $\boldsymbol{n}$ is the unit normal of the plane $\Gamma$, and $0 \leq T_{b} \leq T$. We are free to choose $\boldsymbol{n}$ and $T_{b}$.

Define an operator $K$ for $\boldsymbol{z}=\left(\begin{array}{c}T \\ \boldsymbol{w}\end{array}\right)$ by

$$
K(\boldsymbol{z})=\left(\begin{array}{l}
\boldsymbol{n}^{\dagger} \varphi\left(T_{b}, \boldsymbol{w}\right) \\
\varphi(T, \boldsymbol{w})-\boldsymbol{w}
\end{array}\right)
$$

The zero of $K$, namely $\boldsymbol{z}^{*}$ such that $K\left(\boldsymbol{z}^{*}\right)=0$, gives the solution as $\boldsymbol{z}^{*}=\left(\begin{array}{c}T^{*} \\ \boldsymbol{w}^{*}\end{array}\right)$.

\subsection{Validation of zero point}

The Jacobi matrix $K_{z}$ of $K$ with respect to $z$ is as follows.

$$
K_{z}=\left(\begin{array}{cc}
0 & \boldsymbol{n}^{\dagger} V\left(T_{b}, \boldsymbol{w}\right) \\
\boldsymbol{f}(T, \boldsymbol{w}) & V(T, \boldsymbol{w})-I
\end{array}\right),
$$

Using this form we construct a Newton-like operator $N$ in order to verify the zero $z^{*}$ of $K$.

Compute an approximation $\tilde{K}_{z}$ to $K_{z}$ for $\tilde{\boldsymbol{w}}=0$ and $\tilde{T}$, and define $N$ by

$$
N(\boldsymbol{z})=\boldsymbol{z}-\tilde{K}_{z}^{-1} K(\boldsymbol{z}) .
$$

Take a bounded convex closed set $[Z]=([T],[W])$ constructed by an real interval $[T]$ and an interval vector $[W] \subset \Gamma$ which include the approximation $\tilde{T}$ and $\tilde{\boldsymbol{w}}=\mathbf{0}$, respectively. Then iterate the following.

(1) Check the condition $N([Z]) \subset[Z]$ by interval arithmetic with guaranteed accuracy. If the condition holds, then it is proved that there exists a fixed point of $Q$ within $[Z]$ by Brouwer's fixed point theorem.

(2) If the condition dose not hold, calculate $[Z]:=(1+\varepsilon) N([Z])-\varepsilon N([Z])$ by interval arithmetic and go back to (1). Here $\varepsilon$ is a given small positive number.

Similar to HY method, we use various technique of validated numerics in practical computation.

\subsection{Numerical examples}

The question is how to choose the bordering condition. We want to select appropriate $\boldsymbol{n}$ and $T_{b}$ which give good results. In order to find good bordering, numerical examples are computed for the following 3 cases.
(i) $\boldsymbol{n}=\frac{\boldsymbol{f}(\varphi(\tilde{T}, \tilde{\boldsymbol{w}}))}{\|\boldsymbol{f}(\varphi(\tilde{T}, \tilde{\boldsymbol{w}}))\|}, T_{b}=0$.
(ii) $\boldsymbol{n}=\frac{\boldsymbol{f}(\varphi(\tilde{T}, \tilde{\boldsymbol{w}}))}{\|\boldsymbol{f}(\varphi(\tilde{T}, \tilde{\boldsymbol{w}}))\|}, T_{b}=\tilde{T}$.
(iii) $\boldsymbol{n}=\frac{\boldsymbol{f}(\varphi(\tilde{T} / 2, \tilde{\boldsymbol{w}}))}{\|\boldsymbol{f}(\varphi(\tilde{T} / 2, \tilde{\boldsymbol{w}}))\|}, T_{b}=0$. 


\subsubsection{Lorenz equations}

We treat the following system of three ordinary differential equations.

$$
\left\{\begin{array}{l}
\frac{d x}{d t}=a_{1} x+b_{1}(x+y) z \\
\frac{d y}{d t}=a_{2} y-b_{1}(x+y) z \\
\frac{d z}{d t}=-a_{3} z+(x+y)\left(b_{2} x+b_{3} y\right)
\end{array}\right.
$$

The system is obtained from the Lorenz equations using linear change of variables [3]. We use a set of values of the coefficients corresponding to the values of parameters for which Lorenz system has an unstable closed orbit $[3,4]$ :

$$
\begin{gathered}
a_{1}=9.700378782, b_{1}=-0.227266206, \\
a_{2}=-16.700378782, b_{2}=2.616729797, \\
a_{3}=2.666666667, b_{3}=-1.783396463,
\end{gathered}
$$

An approximate time period and an approximate initial point are

$$
\begin{aligned}
& \tilde{T}=0.6899, \\
& \tilde{\boldsymbol{w}}=\left(\begin{array}{c}
3.500 \\
3.330 \\
27
\end{array}\right),
\end{aligned}
$$

and the divided number $N$ and the degree of Taylor expansion in $C^{0}$ and $C^{1}$ algorithm is 2000 and 4 , respectively.

The results are as follows.

Table I. Iteration number for verification.

\begin{tabular}{ccc}
\hline (i) & (ii) & (iii) \\
\hline 6 & 7 & 6
\end{tabular}

Table II. Radius norm after 7 iterations.

\begin{tabular}{ccc}
\hline (i) & (ii) & (iii) \\
\hline $4.950 \times 10^{-10}$ & $1.211 \times 10^{-9}$ & $5.095 \times 10^{-10}$
\end{tabular}

The number of iterations of condition (i) and (iii) are less than that of (ii). Moreover the obtained radius of the interval vector which includes $\boldsymbol{w}^{*}$ of (i) is less than that of (iii) after 7 iterations. Therefore the condition (i) gives superior results to the condition (ii) and (iii).

\subsubsection{Rössler system}

We treat Rössler system [5],

$$
\left\{\begin{array}{l}
\frac{d x}{d t}=-y-z \\
\frac{d y}{d t}=x+a y \\
\frac{d z}{d t}=b-z(c-x)
\end{array} .\right.
$$

We choose values of parameters as $a=0.2, b=0.2, c=2.2$ with which the system has an attracting limit cycle.

An approximate time period and an approximate initial point are 


$$
\begin{aligned}
& \tilde{T}=5,74, \\
& \tilde{\boldsymbol{w}}=\left(\begin{array}{c}
2.2864 \\
-3.6985 \\
0.1339
\end{array}\right) .
\end{aligned}
$$

and the divided number $N$ and the degree of Taylor expansion in $C^{0}$ and $C^{1}$ algorithm is 500 and 4 , respectively.

The results are as follows.

Table III. Iteration number for verification.

\begin{tabular}{ccc}
\hline (i) & (ii) & (iii) \\
\hline 4 & 9 & 5
\end{tabular}

Table IV. Radius norm after 9 iterations.

\begin{tabular}{ccc}
\hline (i) & (ii) & (iii) \\
\hline $1.006 \times 10^{-7}$ & $1.910 \times 10^{-7}$ & $1.041 \times 10^{-7}$
\end{tabular}

The number of iterations of condition (i) is less than that of (ii) and (iii), and the obtained radius of the interval vector which includes $\boldsymbol{w}^{*}$ of (i) is less than that of (iii) after 9 iterations. Moreover we took 20 points on the approximate trajectory to the limit cycle and applied the bordering methods with conditions (i) and (iii). After 9 iterations, all results succeeded in verification and showed that the radius norms of (i) are smaller than those of (iii). Therefore the condition (i) gives superior results to the condition (ii) and (iii).

\section{Interpretation by HY method}

From the above results, the bordering condition (i) is superior in both cases. In this section we show that the two-point boundary value problem with bordering (i) is equivalent to HY method and this explains the advantage of bordering (i).

Now we start from HY method taking $\gamma$ in $Q$ as $\boldsymbol{n}^{\dagger} \boldsymbol{f}(\boldsymbol{w})$ so that the computation of $T_{1}$ in HY method should be an exact Newton iteration for the time period. Put the difference in Newton iteration as $\Delta \boldsymbol{z}=N(\boldsymbol{z})-\boldsymbol{z}$ then we have

$$
\boldsymbol{z}-P_{\Gamma} Q(\boldsymbol{z})=-\left(I-P_{\Gamma} Q_{z}(\boldsymbol{z})\right) \Delta \boldsymbol{z} .
$$

Thanks to the advice from Kaigaishi and Matsuo [6], we define a matrix $R$ which plays a special role in transformation.

$$
R=\left(\begin{array}{cc}
0 & -\boldsymbol{n}^{\dagger} \\
-\gamma \boldsymbol{n} & P_{w}
\end{array}\right)
$$

Multiply Eq. (4) by $R$ from the left-hand side, then we have

$$
\left(\begin{array}{c}
-\boldsymbol{n}^{\dagger} \boldsymbol{w} \\
\boldsymbol{w}-\varphi(T, \boldsymbol{w})
\end{array}\right)=\left(\begin{array}{cc}
0 & -\boldsymbol{n}^{\dagger} \\
-\boldsymbol{f}(\varphi(T, \boldsymbol{w})) & I-\varphi_{w}(T, \boldsymbol{w})
\end{array}\right) \Delta \boldsymbol{z}
$$

which indicates

$$
K(\boldsymbol{z})=-K_{z} \Delta \boldsymbol{z}
$$

with the bordering condition (i). Since the matrix $R$ is nonsingular in any case, this means that HY method and the bordering method with (i) are equivalent. As the convergence of the time period in HY method would be so fast by virtue of exact Newton method, the convergence of the bordering method with (i) would be also fast. On the other hand, condition (ii) nor (iii) does not have such property. This is the reason why the condition (i) is superior to others. 


\section{Conclusion}

- We discussed numerical verification methods for the existence of a closed orbit of dynamical systems.

- The method of Zgliczyński needs accurate values of return times for each step in iterations, and a bisection method is adopted to obtain them. On the other hand, HY method does not need tight estimation of return times for the initial step and obtain accurate interval values of them through iteration process based on Newton method. From theoretical point of view, HY method might have some advantage to Zgliczyński's method because of the difference of Newton method and bisection method.

- HY method is equivalent to the bordering method with the bordering condition (i) which is very simple.

- The bordering method with (i) is superior to other bordering conditions and this comes from convergence property of HY method.

Additional remarks:

- Newton-Rahpson-Mees method [7] is a variation of the bordering method with condition (i). In this method, Poincaré section is updated in each step. It seems efficient in approximate computation, but would be too heavy for validated computation.

- The projection appeared in HY method plays an important role to treat a basin of a limit cycle with asymptotic stability. This will be shown in our future work.

\section{Acknowledgments}

The authors thank to Professor Matsuo and Mr. Kaigaishi in Tokyo university, Japan, for their important advices and kind suggestions.

\section{References}

[1] P. Zgliczyński, "C $C^{1}$-Lohner algorithm," Found. Comput. Math., vol. 2, pp. 429-465, 2002.

[2] T. Hiwaki and N. Ymamoto, "Nummerical verification for existence of closed orbit in dynamical system," Transaction of Japan Society for Industrial and Applied Mathematics, vol. 22, no. 4, pp. 269-276, 2012, in Japanese

[3] Ja.G. Sinai and E.B. Vul, "Discovery of closed orbits of dynamical systems with the use of computers," Journal of Statistical Physics, vol. 23, no. 1, pp. 22-47, 1980.

[4] M. Yamaguchi, H. Yoshihara, and T. Nishida, "Some remarks on a paper of sinai-vul in 1980," vol. 831, pp. 112-114, 1993, in Japanese

[5] O.E. Rössler, "AN EQUATION FOR CONTINUOUS CHAOS," Physics Letters A, vol. 57, no. 5, pp. 397-398, 1976.

[6] W. Kaigaishi and T. Matsuo, "On Newton-Rahpson-Mees method and HY method," Toyo university, The Meeting of the Union of Research Activity Groups, Japan SIAM, 2013, in Japanese

[7] Alistair I. Mees, Dynamics of Feedback Systems, John Wiley and Sons, New York, 1981.

[8] R. Rihm, "Interval methods for initial value problems in ODEs," Topics in validated computation (ed. by J. Herzberger), Elsevier (North-Holland), Amsterdam, 1994. 